\title{
Kinetics and Mechanism of the Addition of Aliphatic Amines to Transient Silenes
}

\author{
William J. Leigh* and Xiaojing Li \\ Department of Chemistry, McMaster University, 1280 Main Street West, Hamilton, Ontario, Canada L8S 4M1
}

Received: June 8, 2002; In Final Form: November 14, 2002

\begin{abstract}
Primary and secondary amines such as $n$-butyl-, $t$-butyl-, and $N, N$-diethylamine add across the $\mathrm{Si}=\mathrm{C}$ bond of transient silenes such as 1,1-diphenylsilene (1a) and 1,1-bis(4-trifluoromethylphenyl)silene (1b) to yield the corresponding amino(methyl)diarylsilanes as the only products of reaction. The kinetics and mechanism of reaction of these three amines with the two 1,1-diarylsilene derivatives have been studied in hexane, acetonitrile, and tetrahydrofuran (THF) solution by laser flash photolysis techniques, using the corresponding 1,1diarylsilacyclobutanes as photochemical precursors to the silenes. The reactions proceed with clean secondorder kinetics and bimolecular rate constants in excess of $5 \times 10^{8} \mathrm{M}^{-1} \mathrm{~s}^{-1}$ in hexane and $\mathrm{MeCN}$, with $\mathbf{1 b}$ being up to four times more reactive than 1a. Arrhenius plots for reaction in hexane and/or acetonitrile solution show strong curvature over the $0-60^{\circ} \mathrm{C}$ temperature range, consistent with an addition mechanism involving the intermediacy of a zwitterionic silene-amine addition complex, which collapses to product by intramolecular proton transfer from nitrogen to the silenic carbon. The reactions are substantially slower in THF, where rate reductions on the order of 5-10-fold and 50-70-fold are observed for $\mathbf{1 a}$ and $\mathbf{1 b}$, respectively, as compared to $\mathrm{MeCN}$ solution. This is due to the effects of complexation of the silenes with the ether solvent, the equilibrium constant for which enters the expression for the reaction rate constant in the complexing solvent. In contrast to the situation in hexane and $\mathrm{MeCN}$, Arrhenius plots for reaction of $n$ - $\mathrm{BuNH}_{2}$ with $\mathbf{1 a}, \mathbf{b}$ in THF solution are linear and lead to positive activation energies. Addition of amines in THF solution is proposed to occur predominantly via the free silenes but with minor contributions from a pathway involving nucleophilic displacement of the solvent from the silene-solvent complex.
\end{abstract}

\section{Introduction}

Silenes react regiospecifically with nucleophiles such as water, alcohols, carboxylic acids, alkoxysilanes, and amines by (1,2)-addition across the $\mathrm{M}=\mathrm{C}$ bond. ${ }^{1-9}$ The mechanism of alcohol addition has been extensively studied and is particularly well-understood (eq 1). ${ }^{10,11}$ It is initiated by reversible nucleophilic attack at silicon to form a zwitterionic complex, ${ }^{1,12}$ which proceeds to product by two competing, rate-limiting proton transfer pathways-one intramolecular and one involving a second molecule of alcohol (see eq 1). ${ }^{13-15}$ The two proton transfer pathways have different stereo- and regiochemical consequences; the intramolecular pathway leads to stereoselective syn $(1,2)$-addition, while the bimolecular pathway leads to overall trans addition or the formation of $(1,4)$ - and $(1,6)$ addition products if a polyenic substituent is present on the silenic carbon. ${ }^{14}$ Time-resolved spectroscopic studies have provided kinetic evidence for the two proton transfer pathways in selected cases, in the form of a mixed first- and secondorder dependence of the rate on alcohol concentration. ${ }^{14,16,17}$ However, most of the transient silenes that have been studied to date are sufficiently reactive toward alcohols and water that only the second-order reaction pathway can be detected by these methods. ${ }^{9}$ The 1,1-diarylsilene derivatives $\mathbf{1 a}-\mathbf{e}$ are examples of silenes that show such behavior and have proven extremely useful in delineating the quantitative aspects of silene reactivity

* To whom correspondence should be addressed. E-mail: leigh@ mcmaster.ca. toward alcohols ${ }^{18,19}$ and various other oxygen-centered nucleophiles. ${ }^{9}$
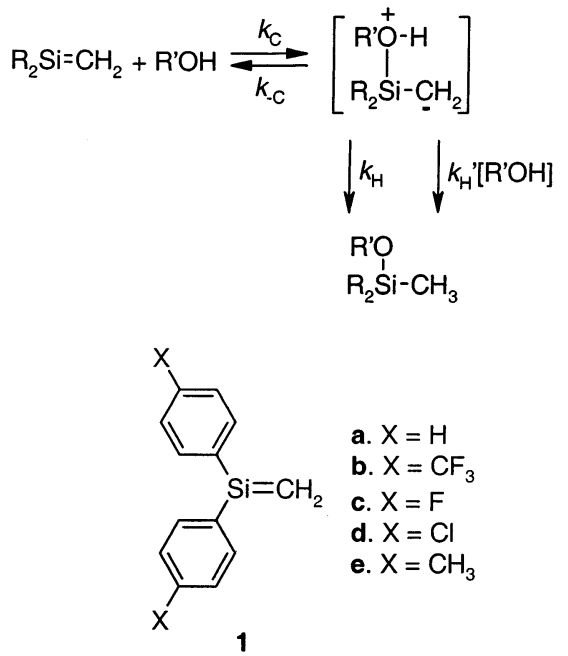

a. $X=H$

b. $\mathrm{X}=\mathrm{CF}_{3}$

c. $X=F$

d. $\mathrm{X}=\mathrm{Cl}$

e. $\mathrm{X}=\mathrm{CH}_{3}$

The reaction of silenes with nitrogen-centered nucleophiles is also well-known, ${ }^{1,3,8}$ but no detailed absolute kinetic studies have yet been reported. ${ }^{7}$ More than 15 years ago, Wiberg and co-workers published the results of competition experiments on the addition of a comprehensive series of reagents to silene $\mathbf{2}$ and showed that primary amines are slightly more reactive than aliphatic alcohols toward addition to the $\mathrm{Si}=\mathrm{C}$ bond of this compound. ${ }^{1}$ Preliminary kinetic experiments in our laboratory 
on the addition of $n$-butylamine $\left(n-\mathrm{BuNH}_{2}\right)$ to 1,1 -diphenylsilene (1a) have shown this to be true of this silene as well. ${ }^{7}$ This and Wiberg and co-workers' characterization of metastable complexes of tertiary aliphatic amines with $\mathbf{2}^{2}$ and the related silene $\mathbf{3}^{4}$ suggest that as with alcohol addition, the reaction with primary amines is initiated by nucleophilic attack at silicon, followed by proton transfer from nitrogen to carbon.<smiles>CC(C)=C(C)C</smiles>

2

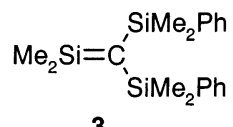

3
In this paper, we report the results of a laser flash photolysis study of the kinetics of addition of primary and secondary amines to the silicon-carbon double bonds of 1a and 1,1-bis(4-trifluoromethylphenyl)silene (1b) in hexane, acetonitrile (MeCN), and tetrahydrofuran (THF) solution, with a view of fully defining the kinetics and mechanism for the addition of aliphatic amines to transient silenes.

\section{Results}

Steady state photolysis (254 nm) of deoxygenated $0.02-0.08$ $\mathbf{M}$ solutions of the 1,1-diarylsilacyclobutanes $\mathbf{4 a}, \mathbf{b}$ in hexane or cyclohexane- $d_{12}$ containing a ca. $10 \%$ molar excess of $n$-BuNH 2 , tert-butylamine $\left(t-\mathrm{BuNH}_{2}\right)$, or $N, N$-diethylamine $\left(\mathrm{Et}_{2}-\right.$ $\mathrm{NH}$ ) afforded (along with ethylene) the corresponding aminosilanes 5-7 as the major nitrogen-containing products (eq 2). These are the products expected from $(1,2)$-addition of the amines to silenes $\mathbf{1 a}$ and $\mathbf{1 b}$, respectively. The secondary aminosilanes formed from $n-\mathrm{BuNH}_{2}$ and $t-\mathrm{BuNH}_{2}(\mathbf{5 a}, \mathbf{b}$ and $\mathbf{6 a}, \mathbf{b})$ are evidently much less reactive than the primary amines from which they are derived, as the photolyses could be carried out to quite high conversions, with little or no evidence for the formation of secondary products corresponding to addition of 5 or 6 to 1 .

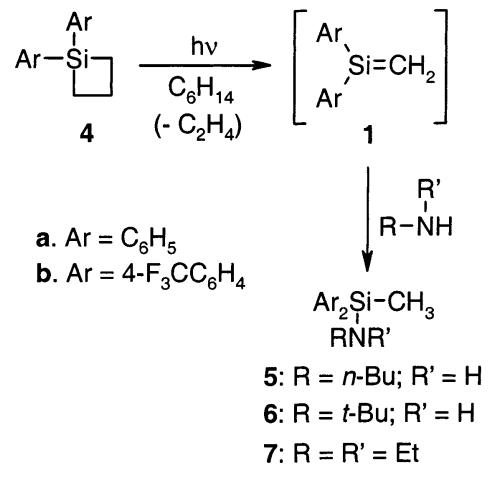

Laser flash photolysis of continuously flowing solutions of 4a and $4 \mathbf{b}(\sim 0.004 \mathrm{M})$ in dry hexane or $\mathrm{MeCN}\left(\left[\mathrm{H}_{2} \mathrm{O}\right] \leq 10^{-4}\right.$ M) gave rise to transient absorptions $\left(\lambda_{\max }=325 \mathrm{~nm}\right)$ assignable to $\mathbf{1 a}$ and $\mathbf{1 b}$, respectively, as previously reported. ${ }^{18,20,21}$ The two silenes exhibit lifetimes of $>5 \mu \mathrm{s}$ in hexane and $1-4 \mu \mathrm{s}$ in acetonitrile under these conditions and decay with mixed firstand second-order kinetics, with 1a being the longer-lived of the two. Much different behavior is observed in THF solution, where the lifetimes of the two silenes are longer, $\mathbf{1 b}$ is the longer-lived of the two, and their spectra are broadened and/or red-shifted as compared to hexane solution because of complexation with the ether solvent. ${ }^{19}$ As reported previously, ${ }^{19}$ the spectrum of 1a varies over the $0-60{ }^{\circ} \mathrm{C}$ temperature range, exhibiting $\lambda_{\max }=325$ and $350 \mathrm{~nm}$ at the high and low ends of this temperature range, respectively. Complexation is stronger
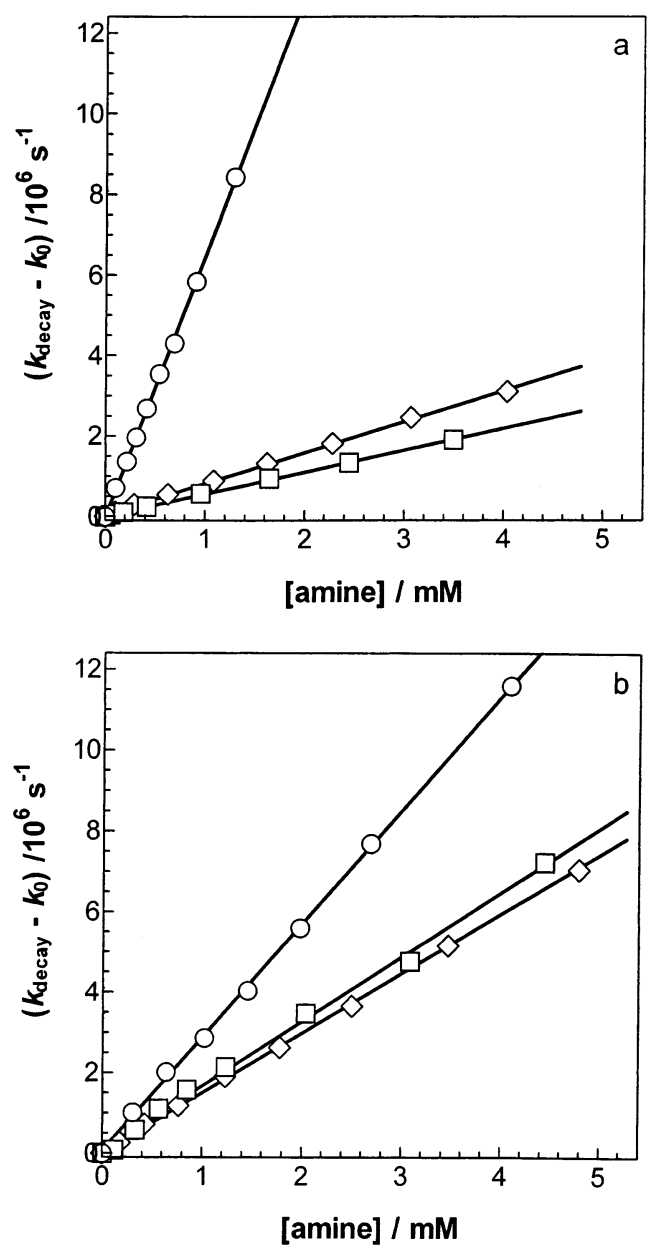

Figure 1. Plots of $\left(k_{\text {decay }}-k_{0}\right)$ vs [amine] for quenching of 1a by $n-\mathrm{BuNH}_{2}(\mathrm{O}), t-\mathrm{BuNH}_{2}(\square)$, and $\mathrm{Et}_{2} \mathrm{NH}(\diamond)$ in (a) hexane and (b) acetonitrile solution at $23{ }^{\circ} \mathrm{C}$.

for the more electrophilic silene (1b), which results in a greater spectral shift $\left(\lambda_{\max }=375 \mathrm{~nm}\right)$, only slight variations in the spectrum with temperatures between 0 and $60{ }^{\circ} \mathrm{C}$, and a more pronounced lengthening in lifetime as compared to 1a. Under these conditions, the lifetimes of $\mathbf{1 a}$ and $\mathbf{1 b}$ are limited by the rates of dimerization and reaction with trace amounts of water.

The lifetimes of $\mathbf{1 a}, \mathbf{b}$ were shortened upon addition of $n$ - $\mathrm{BuNH}_{2}, t-\mathrm{BuNH}_{2}$, or $\mathrm{Et}_{2} \mathrm{NH}$ to the solutions. The decays followed clean pseudo-first-order kinetics in the presence of amine, with individual lifetimes that were independent of monitoring wavelength throughout the $310-400 \mathrm{~nm}$ range in each solvent. The decay rate constants $\left(k_{\text {decay }}\right)$ varied linearly with amine concentration according to eq 3 , where $k_{\text {amine }}$ is the second-order rate constant for reaction of $\mathbf{1} \mathbf{a}, \mathbf{b}$ with amine and $k_{0}$ is the pseudo-first-order rate constant for silene decay in the absence of amine. For example, Figure 1 shows plots of the corrected pseudo-first-order rate constants for decay of $\mathbf{1 a}\left(k_{\text {decay }}\right.$ $\left.-k_{0}\right)$ as a function of amine concentration in hexane and acetonitrile solution (the corresponding plots for silene $\mathbf{1 b}$ are available as Supporting Information). Table 1 lists the absolute second-order rate constants obtained from these experiments.

$$
k_{\text {decay }}=k_{0}+k_{\text {amine }}[\text { amine }]
$$

Addition of up to ca. $0.01 \mathrm{M}$ triethylamine caused no change in the lifetime or absorption spectrum of either silene in hexane solution. Only the initial transient $\triangle O D$ values varied as a function of amine concentration, becoming increasingly smaller 
TABLE 1: Absolute Rate Constants (in Units of $10^{9} \mathrm{M}^{-1}$ $\mathrm{s}^{-1}$ ) for Reaction of Aliphatic Amines with $1 \mathrm{a}$ and $1 \mathrm{~b}$ in Hexane, MeCN, and THF Solution at $23^{\circ} \mathrm{C}^{a}$

\begin{tabular}{llccc}
\hline & \multicolumn{4}{c}{$k_{\text {amine }}\left(10^{9} \mathrm{M}^{-1} \mathrm{~s}^{-1}\right)$} \\
\cline { 2 - 5 } \multicolumn{1}{c}{ silene } & \multicolumn{1}{c}{ amine } & \multicolumn{1}{c}{ hexane } & $\mathrm{MeCN}$ & $\mathrm{THF}$ \\
\hline $\mathrm{Ph}_{2} \mathrm{Si}=\mathrm{CH}_{2}$ & $n-\mathrm{BuNH}_{2}$ & $6.49 \pm 0.12$ & $2.81 \pm 0.06$ & $0.32 \pm 0.04$ \\
$(\mathbf{1 a})$ & $t-\mathrm{BuNH}_{2}$ & $0.54 \pm 0.01$ & $1.59 \pm 0.07$ & $0.150 \pm 0.004$ \\
& $\mathrm{Et}_{2} \mathrm{NH}$ & $0.77 \pm 0.02$ & $1.48 \pm 0.06$ & $0.148 \pm 0.005$ \\
$\left(4-\mathrm{CF}_{3} \mathrm{C}_{6} \mathrm{H}_{4}\right)_{2}-$ & $n-\mathrm{BuNH}_{2}$ & $10.6 \pm 0.1$ & $3.28 \pm 0.06$ & $0.094 \pm 0.003$ \\
$\mathrm{Si}=\mathrm{CH}_{2}$ & $t-\mathrm{BuNH}_{2}$ & $2.03 \pm 0.04$ & $2.06 \pm 0.11$ & $0.032 \pm 0.001$ \\
$(\mathbf{1 b})$ & $\mathrm{Et}_{2} \mathrm{NH}$ & $3.33 \pm 0.10$ & $1.46 \pm 0.11$ & $0.020 \pm 0.001$
\end{tabular}

${ }^{a}$ Errors are reported as $\pm 2 \sigma$ from least squares analyses of $k_{\text {decay }}$ vs [amine] data according to eq 3 .
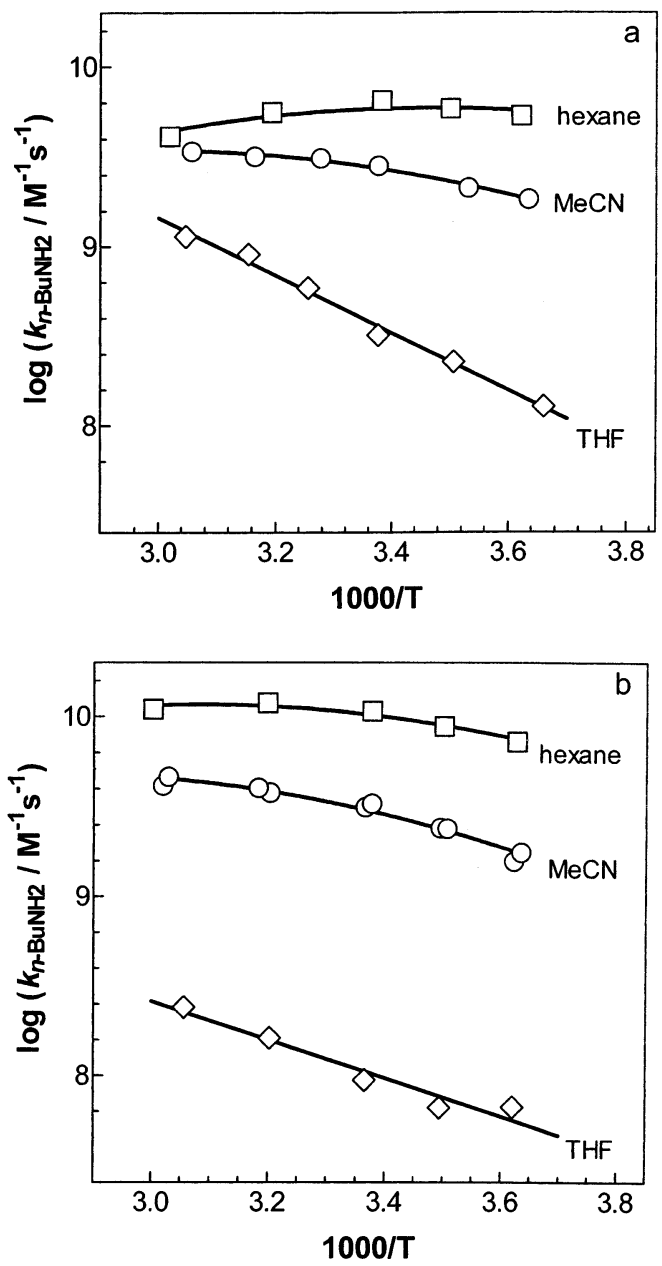

Figure 2. Arrhenius plots for reaction of silenes $\mathbf{1 a}(\mathrm{a})$ and $\mathbf{1 b}(\mathrm{b})$ with $n$ - $\mathrm{BuNH}_{2}$ in hexane $(\square)$, acetonitrile $(\bigcirc)$, and THF $(\diamond)$ solution.

as the amine concentration was increased due to competing absorption of the excitation light by the amine. It was thus not possible to carry out experiments at amine concentrations high enough to enable discrete spectroscopic observation of sileneamine complexes.

Absolute rate constants for reaction of $\mathbf{1 a}, \mathbf{b}$ with $n-\mathrm{BuNH}_{2}$ were determined at several temperatures between 0 and $60^{\circ} \mathrm{C}$ in the three solvents and are shown as Arrhenius plots in Figure 2. Similar experiments were carried out for the reactions of $\mathbf{1 a}, \mathbf{b}$ with $t-\mathrm{BuNH}_{2}$ and $\mathrm{Et}_{2} \mathrm{NH}$ in acetonitrile, the results of which are shown in Figure 3 along with those for $n-\mathrm{BuNH}_{2}$ from Figure 2. For comparison, the temperature dependencies of the diffusional rate constants in acetonitrile (calculated using the standard Debye equation $\left(k_{\mathrm{diff}}=8 R T / 3000 \eta\right)$ and published temperatureviscosity data $^{22}$ ) are also shown in the plots of Figure 3 . The
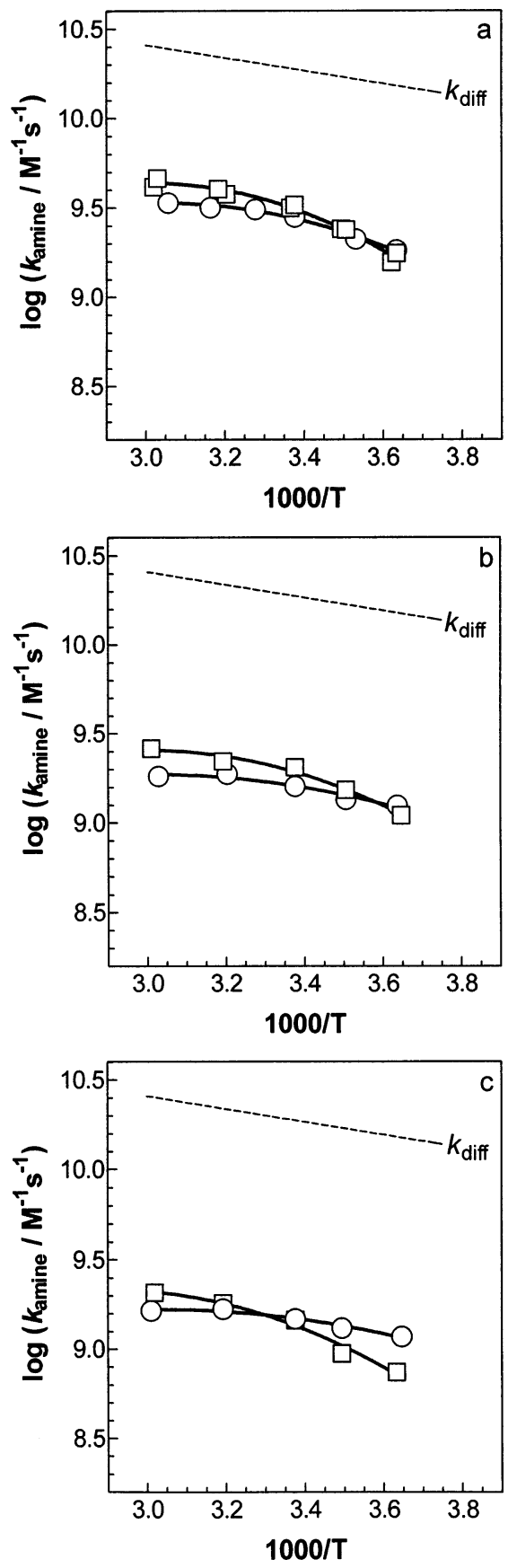

Figure 3. Arrhenius plots for reaction of silenes 1a $(O)$ and $\mathbf{1 b}(\square)$ with $n$-butylamine (a), tert-butylamine (b), and $N, N$-diethylamine (c) in dry acetonitrile solution.

Arrhenius plots for reaction of $\mathbf{1 a}, \mathbf{b}$ with the three amines in hexane and/or acetonitrile solution all show distinct curvature over the $0-60{ }^{\circ} \mathrm{C}$ temperature range. In contrast, those for reaction of $n-\mathrm{BuNH}_{2}$ in THF are linear; least squares analyses afforded the Arrhenius parameters $E_{\mathrm{a}}=7.3 \pm 0.6 \mathrm{kcal} / \mathrm{mol}$ and $\log \left(\mathrm{A} / \mathrm{M}^{-1} \mathrm{~s}^{-1}\right)=14.0 \pm 0.5\left(r^{2}=0.9914\right)$ in the case of 1a and $E_{\mathrm{a}}=4.9 \pm 1.3 \mathrm{kcal} / \mathrm{mol}$ and $\log \left(\mathrm{A} / \mathrm{M}^{-1} \mathrm{~s}^{-1}\right)=11.6 \pm 1.0$ $\left(r^{2}=0.9476\right)$ in the case of $\mathbf{1 b}$.

\section{Discussion}

The 1,1-diarylsilenes 1a,b are both exceedingly electrophilic and react rapidly with the three amines studied in this work. The nonhindered primary amine $n-\mathrm{BuNH}_{2}$ reacts somewhat faster than its more hindered isomers $t-\mathrm{BuNH}_{2}$ and $\mathrm{Et}_{2} \mathrm{NH}$, especially in hexane solution. This can probably be ascribed to 
steric effects, given that the (nonaqueous) $\mathrm{p} K_{\mathrm{a}}$ values of the three amines are essentially identical. ${ }^{23}$ Silene $\mathbf{1 b}$ is consistently a factor of ca. 2-4 times more reactive than 1a in hexane solution toward each amine studied, as we have found previously for the reactions of these two silenes with oxygen-centered nucleophiles. ${ }^{18}$ This indicates that the Hammett $\rho$-value for amine addition to 1,1-diarylsilenes is small but positive, consistent with a reaction mechanism analogous to that for oxygen-centered nucleophiles. ${ }^{9}$ This mechanism is shown in eq 4 , while eq 5 gives the expression for the overall rate constant for reaction $\left(k_{\text {amine }}\right)$, derived using the steady state assumption for the intermediate complex.

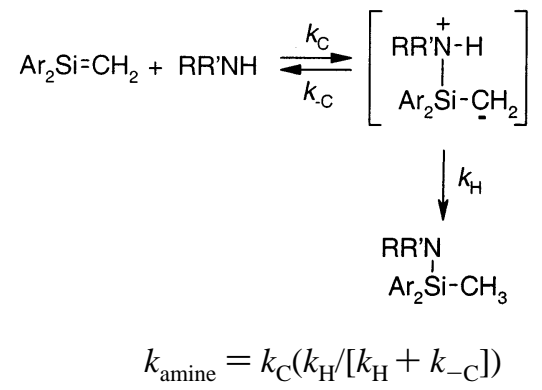

More evidence for this mechanism is provided by the temperature dependencies of the absolute rate constants for reaction of the two silenes with $n-\mathrm{BuNH}_{2}$ in hexane and $\mathrm{MeCN}$ solution (Figure 2). The nonlinear variations in $\log k_{\text {amine }}$ with $T^{-1}$ result from the superposition of a positive temperature dependence of $k_{\mathrm{C}}$ on a negative temperature dependence of the complex partitioning ratio, $k_{\mathrm{H}} /\left(k_{\mathrm{H}}+k_{-\mathrm{C}}\right)$, which occurs because the reaction is strongly exothermic and the rate constant for the product-forming step $\left(k_{\mathrm{H}}\right)$ is dominated by entropic factors. ${ }^{9}$ The temperature dependence of $k_{\text {amine, }}$ as defined by this mechanism, is given explicitly by eq 6 , where $A_{\mathrm{C}}$ and $E_{\mathrm{C}}$ are the Arrhenius preexponential factor and activation energy for formation of the intermediate complex, $A_{-\mathrm{C}}$ and $A_{\mathrm{H}}$ are the preexponential factors for reversion of the complex to starting materials and collapse to product, respectively, and $\Delta E_{-\mathrm{C}, \mathrm{H}}$ ( $\left.=E_{-\mathrm{C}}-E_{\mathrm{H}}\right)$ is the difference in activation energies for the two processes. Integration of the logarithmic form of this expression with respect to $T^{-1}$ leads to the prediction that $k_{\text {amine }}$ goes through a maximum value (i.e., $E_{\mathrm{a}}$ overall $=0$ ) at the temperature where the partitioning ratio is equal to the quantity $\left(1-E_{\mathrm{C}} / \Delta E_{-\mathrm{C}, \mathrm{H}}\right){ }^{24}$ The Arrhenius plot exhibits a positive slope (negative $E_{\mathrm{a}}$ ) over the temperature range where the partitioning ratio is less than this value and a negative slope (positive $E_{\mathrm{a}}$ ) over the range where it exceeds it.

$k_{\text {amine }}=\left[A_{\mathrm{C}} \exp \left(-E_{\mathrm{C}} / R T\right)\right] /\left[1+\left(A_{-\mathrm{C}} / A_{\mathrm{H}}\right) \exp \left(-\Delta E_{-\mathrm{C}, \mathrm{H}} / R T\right)\right]$

While the solid lines in the plots for quenching in hexane and acetonitrile solution shown in Figures 2 and 3 represent the best fits of the experimental data to eq 6, the four Arrhenius parameters obtained from the analyses have extremely large errors associated with them, not unexpectedly considering the small number of data points obtained and the relatively narrow temperature range over which they were determined. Accordingly, we can offer only a few qualitative comments on the data at the present time. First, the data show clearly that the overall rate constants for reaction ( $k_{\text {amine }}$ ) of $\mathbf{1 a}$ and $\mathbf{1 b}$ with $n-\mathrm{BuNH}_{2}$ in hexane take on maximum values of $(6-7) \times 10^{9} \mathrm{M}^{-1} \mathrm{~s}^{-1}$ and ca. $1.2 \times 10^{10} \mathrm{M}^{-1} \mathrm{~s}^{-1}$, respectively, values that are within a factor of 3 and 2 (respectively) of the diffusional rate constants, calculated at the corresponding temperatures using the standard
Debye equation and published viscosities. ${ }^{22}$ This indicates that the initial complexation of the two silenes with the amine proceeds with rate constants $\left(k_{\mathrm{C}}\right)$ that are similar to one another and close or equal to the diffusion-controlled limit. Second, the maximum rate constant for reaction of the more reactive derivative (1b) in hexane solution is attained at a higher temperature $\left(40-50^{\circ} \mathrm{C}\right)$ than that for the less reactive derivative $\left(1 \mathrm{a} ; 15-25^{\circ} \mathrm{C}\right)$. This suggests that at any given temperature within the range studied, collapse of the intermediate complex to product is more efficient (and hence less kinetically significant) for the more reactive derivative $\mathbf{1 b}$, assuming similar activation parameters for the complexation step in the two derivatives. $^{25}$ On the basis of our earlier studies of the complexation of these two compounds with THF, ${ }^{19}$ we suspect that this is due mainly to $k_{-\mathrm{C}}$ being smaller for the more reactive derivative. Unfortunately, attempts to detect complexes of $\mathbf{1 a}, \mathbf{b}$ with triethylamine were unsuccessful due to light absorption problems with solutions containing more than $0.01 \mathrm{M}$ amine, so we are unable to compare the quantitative details of tertiary amine complexation with those of an oxygen-centered nucleophile such as THF. Nevertheless, the trends observed here in the temperature dependencies of the reactions of $\mathbf{1 a}, \mathbf{b}$ with aliphatic amines are similar to those reported previously by us for the reactions of these silenes with aliphatic alcohols, ${ }^{18}$ acetic acid, ${ }^{18}$ acetone, ${ }^{21}$ and methoxytrimethylsilane. ${ }^{20}$ In the latter case, the electrophilic component of the trapping agent is the trimethylsilyl group, rather than a proton.

Figure 4 shows a comparison of the Arrhenius data for reaction of $\mathbf{1 a}, \mathbf{b}$ with $n-\mathrm{BuNH}_{2}$ in acetonitrile to our previously reported data for addition of $\mathrm{MeOH}, \mathrm{AcOH}$, and acetone to the same two silenes in the same solvent. ${ }^{18,21}$ It is interesting to note that on a qualitative level, the kinetics of amine addition exhibit characteristics most like those of acetic acid addition; both reactions exhibit positive temperature dependencies over the $0-60{ }^{\circ} \mathrm{C}$ temperature range, relatively small variations in rate with ring substituents in the silene, and overall rate constants in excess of $10^{9} \mathrm{M}^{-1} \mathrm{~s}^{-1}$. The positive temperature dependencies indicate that the kinetics of both reactions are dominated by the initial complexation step and thus that the partitioning of the intermediate complexes strongly favors product formation in both cases (i.e., $k_{\mathrm{H}}>k_{-\mathrm{C}}$ in eq 5). The data for 1a suggest that the rate constant for the initial complexation step is somewhat higher for the amine, as would be expected considering its higher nucleophilicity as compared to the carboxylic acid. The difference is small, however, and narrows further as the electrophilicity of the silene is increased through $\mathrm{CF}_{3}$ substitution in the aryl rings. While substantial differences would be predicted in the absolute magnitudes of the rate constants for reversion of the intermediate complex to reactants and collapse to product ( $k_{-\mathrm{C}}$ and $k_{\mathrm{H}}$, respectively) for amine vs carboxylic acid addition, because of the dramatically different nucleophilicities and proton acidities of the two reagents, these differences are masked by the fact that it is their relative magnitudes that determine the overall rate constant for reaction and the form of its temperature dependence.

The data of Figure 2 show that in acetonitrile solution, the reactivities of $\mathbf{1 a}, \mathbf{b}$ with $n-\mathrm{BuNH}_{2}$ are lower and the apexes of the Arrhenius plots are shifted toward higher temperatures as compared to the situation in hexane solution. This behavior is also analogous to what has been observed previously for the addition of oxygen-centered nucleophiles to these two silenes. ${ }^{15,18,21}$ In the case of oxygen-centered nucleophiles, we have recently shown that these trends are due, at least to some extent, to the effects of weak complexation of the free silene 

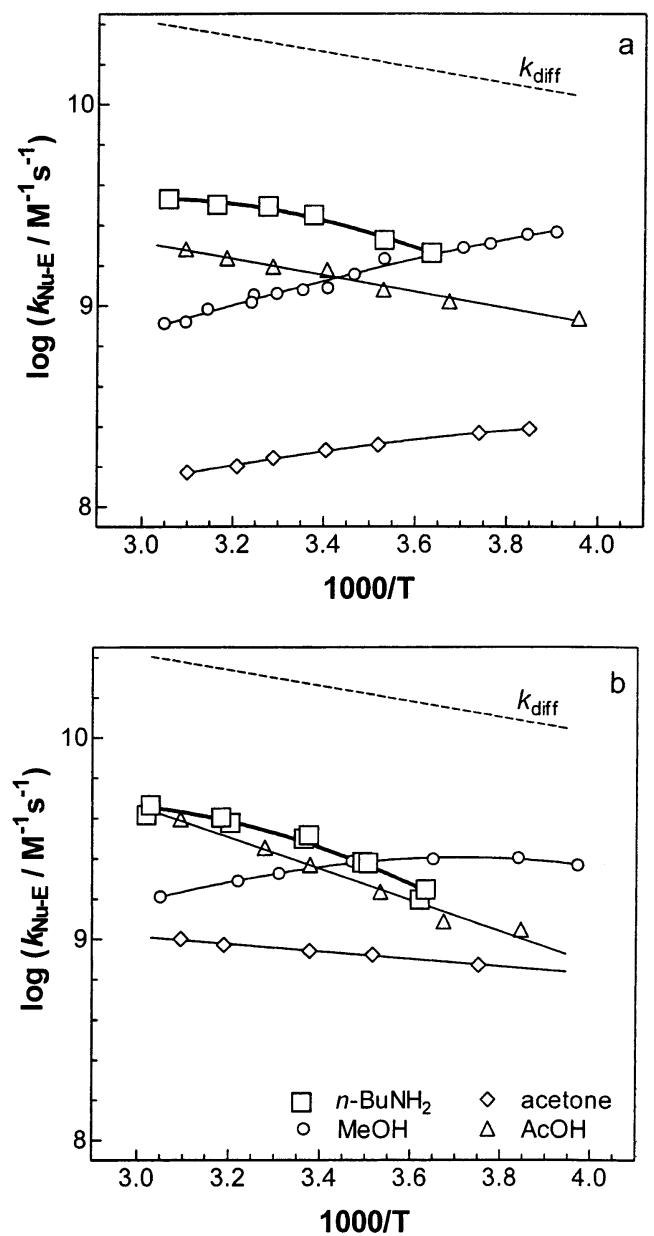

Figure 4. Arrhenius plots for reaction of 1a (a) and $\mathbf{1 b}$ (b) with $n$ - $\mathrm{BuNH}_{2}(\square), \mathrm{MeOH}(O)$, acetone $(\diamond)$, and $\mathrm{AcOH}(\Delta)$ in acetonitrile solution. Data for $\mathrm{MeOH}$ and $\mathrm{AcOH}$ : Reprinted from ref 18. Copyright 1997 National Research Council of Canada. Data for acetone: Reprinted from ref 21. Copyright 1998 American Chemical Society.

with the nitrile solvent. ${ }^{19}$ Such complexation reduces overall reactivity by lowering the concentration of free silene available for reaction with the nucleophile; furthermore, because the equilibrium constant for solvent complexation increases with decreasing temperature, the overall $E_{\mathrm{a}}$ values for reaction in the nitrile solvent are shifted to more positive values relative to hexane solution because of the superposition of the temperature dependence of this (nonproductive) phenomenon on the true temperature dependence of the reaction. ${ }^{19}$ This effect provides an explanation for the small but nonetheless interesting variations in the relative reactivities of $\mathbf{1 a}, \mathbf{b}$ with the three amines in acetonitrile solution as a function of temperature (Figure 3); $\mathbf{1 b}$ is the more reactive of the two silenes at high temperatures, but the difference decreases or even inverts as the temperature is lowered. Even larger changes in the relative reactivity of the two silenes are effected in THF solution, where $\mathbf{1 b}$ is substantially less reactive than 1a throughout the entire temperature range studied. The ether solvent is much less polar than acetonitrile but more strongly complexing, particularly with silicon electrophiles.

Solvent complexation alters the mechanism for reaction of silenes relative to those in noncomplexing solvents as described in eq 7, where $K_{\text {Solv }}$ is the equilibrium constant for solvent complexation, and $k_{1}$ and $k_{1-\text { Solv }}$ are the rate constants for reaction of the nucleophile $(\mathrm{Nu}-\mathrm{E})$ with the free and complexed forms of the silene, respectively. ${ }^{19,26}$ In the general situation where both the free and the complexed forms react, the overall second-order rate constant for reaction in the complexing solvent $\left(k_{\mathrm{Nu}-\mathrm{E}^{\mathrm{S}}}^{\mathrm{S} l v}\right)$ is given by the equivalent expressions of eqs 8 and 9, where $F_{1}$ and $F_{1-\text { Solv }}$ are, respectively, the fractional concentrations of free and complexed silene present in solution. If the complexed form of the silene is substantially less reactive than the uncomplexed form, as might be expected for particularly nonacidic nucleophilic reagents, then the expression for the overall rate constant for reaction simplifies to that of eq 10 . This limiting situation is the one that will lead to the largest (positive) shift in the observed $E_{\mathrm{a}}$ for reaction in a complexing solvent, as compared to its value in a noncomplexing solvent of similar bulk polarity. ${ }^{19}$ For reactions that exhibit very small or negative intrinsic activation energies in noncomplexing solvents, the temperature dependence for reaction in a complexing medium will be dominated by the complexation phenomenon itself; this probably accounts for the observation of (apparent) preexponential factors that approach or exceed those of diffusion. For two silenes of differing intrinsic electrophilicities, and hence different equilibrium constants for complexation with a nucleophilic solvent, this can also lead to an inversion in their relative reactivities in a strongly complexing solvent as compared to the situation in non- or weakly complexing solvents.

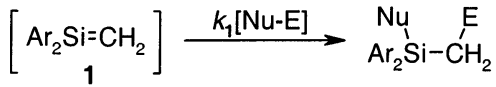

$$
\begin{aligned}
& \text { 4. } K_{\text {Solv }}[\text { Solv] }
\end{aligned}
$$

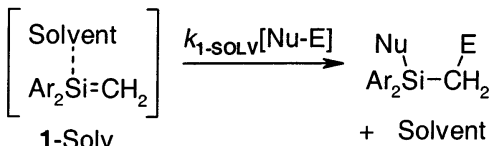

$$
\begin{aligned}
& k_{\mathrm{Nu}-\mathrm{E}}^{\text {Solv }}=F_{1} k_{1}+F_{1-\text { Solv }} k_{1-\text { Solv }} \\
& k_{\mathrm{Nu}-\mathrm{E}}^{\mathrm{Solv}}=\frac{1}{\left(1+K_{\mathrm{Solv}}[\mathrm{Solv}]\right)} k_{1}+\frac{K_{\mathrm{Solv}}[\mathrm{Solv}]}{\left(1+K_{\mathrm{Solv}}[\mathrm{Solv}]\right)} k_{1-\mathrm{Solv}} \\
& k_{\mathrm{Nu}-\mathrm{E}}^{\mathrm{Solv}} \sim F_{1} \cdot k_{1}=\frac{1}{\left(1+K_{\mathrm{Solv}}[\mathrm{Solv}]\right)} \cdot k_{1}
\end{aligned}
$$

The kinetic data for the reactions of $\mathbf{1 a}, \mathbf{b}$ with the three amines in THF solution are fully consistent with this analysis and show trends similar to those reported previously for reaction of $\mathbf{1 a}, \mathbf{b}$ with acetone, another nucleophilic silene trap of especially low Bronsted acidity. ${ }^{19}$ As with acetone addition, the activation energies for reaction of $\mathbf{1 a}, \mathbf{b}$ with $n-\mathrm{BuNH}_{2}$ are shifted to substantially more positive values and the relative reactivities of the two silenes are inverted in THF solution as compared to hexane or MeCN. Moreover, the magnitude of the differences in reactivity of $\mathbf{1 a}, \mathbf{b}$ in $\mathrm{THF}$ relative to $\mathrm{MeCN}$ is similar to that reported previously for acetone addition, ${ }^{19}$ consistent with the fact that amines also fit into the general category of weakly acidic nucleophiles that in THF solution, react exclusively (or almost so; vide infra) via the free silene. This is illustrated more clearly in Figure 5, which shows the Arrhenius plots for the addition of $n-\mathrm{BuNH}_{2}$ to $\mathbf{1 a}, \mathbf{b}$ in THF solution, along with our previously reported data for acetone and $\mathrm{MeOH}$ addition. ${ }^{19}$ As can be seen in the figure, both acetone and $n-\mathrm{BuNH}_{2}$ addition exhibit similarly large, positive activation energies in THF, which has been shown in the former case ${ }^{19}$ to mainly reflect the temperature dependence of $K_{\text {Solv }}$ (or $F_{1}$ in eq 10). On the other hand, reaction with $\mathrm{MeOH}$ proceeds with similar absolute 

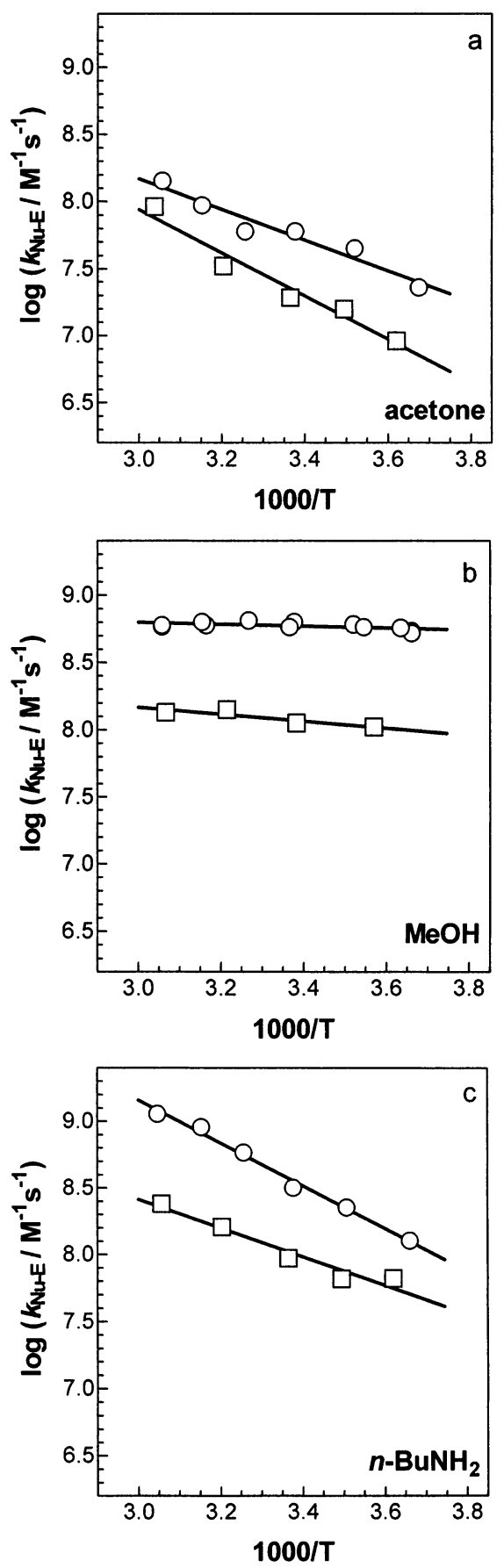

Figure 5. Arrhenius plots for reaction of silenes $\mathbf{1 a}(O)$ and $\mathbf{1 b}(\square)$ with acetone (a), $\mathrm{MeOH}$ (b), and $n$-butylamine (c) in dry THF solution. Panels a and b: Reprinted from ref 19. Copyright 2002 American Chemical Society.

rate constants to that with $n-\mathrm{BuNH}_{2}$ but exhibits almost no variation in rate with temperature. This has been proposed to be due to the intervention of a second reaction pathway, involving reaction of the alcohol with the complexed form of the silene via initial protonation at carbon, that competes with the "normal" mechanism involving reaction with the free silene via initial nucleophilic attack at silicon. ${ }^{19}$ The smaller positive activation energies result from the fact that this pathway should increase in importance with decreasing temperature, as the solvent complexation equilibrium increasingly favors the silenesolvent complex over the free silene. It should be especially important in the case of $\mathbf{1 b}$, which complexes more strongly with the ether solvent. This second pathway is sufficiently minor in the case of alcohol addition that it does not alter the extent to which the relative reactivities of $\mathbf{1 a}, \mathbf{b}$ are inverted in the complexing solvent as compared to noncomplexing solvents. It does appear to be more dominant with reasonably acidic nucleophilic traps such as acetic acid, and as a result, there is very little net change in the relative reactivities of $\mathbf{1 a}, \mathbf{b}$ toward this reagent in THF solution as compared to the more weakly complexing solvent $\mathrm{MeCN}$.

While the idea that silene-THF complexes should be prone to reaction with particularly acidic nucleophilic traps is a reasonable one that has some experimental support, one might also expect them to be reactive toward particularly strong nucleophiles, yielding the same nucleophile-silene addition complex as involved in the reaction with the free silene but by a mechanism involving $\mathrm{S}_{\mathrm{N}} 2$ displacement of the solvent in the first step of the reaction. This could account for the fact that the activation energy for reaction of $\mathbf{1 b}$ with $n-\mathrm{BuNH}_{2}$ in THF is smaller than that for reaction of $\mathbf{1 a}$, in contrast to the opposite trend in activation energies that is observed for reaction of the two silenes with acetone in this solvent (Figure 5). Because reaction by this pathway can be expected to be intrinsically much slower than that involving the free silenes, it can be expected to exhibit a substantial substituent effect, with a reasonably large, positive Hammett $\rho$-value. Thus, it is expected to be more important with $\mathbf{1 b}$ than with $\mathbf{1 a}$, and because the extent to which it contributes to the overall rate constant for reaction will increase as the fractional concentration of complexed silene increases (i.e., with decreasing temperature), it could account for $\mathbf{1 b}$ exhibiting a smaller positive activation energy than 1a.

\section{Summary and Conclusions}

The transient 1,1-diarylsilenes $\mathbf{1 a}, \mathbf{b}$ react with aliphatic amines by regiospecific $(1,2)$-addition, with absolute rate constants within a factor of 10 of the diffusional rate constants in hexane and acetonitrile solution over the $0-60{ }^{\circ} \mathrm{C}$ temperature range. Similar reactivities are observed toward more hindered primary amines and secondary amines under similar conditions. The Arrhenius plots for these reactions show strong curvature over this temperature range, which is consistent with a stepwise reaction mechanism analogous to that for addition of oxygencentered nucleophiles such as methanol: reaction begins by reversible attack of the neutral nucleophile at silicon to yield a zwitterionic addition complex, which proceeds to product by intramolecular proton transfer from the nucleophilic site to the silenic carbon.

Positive activation energies for reaction are observed for the reaction of $n-\mathrm{BuNH}_{2}$ with $\mathbf{1 a}, \mathbf{b}$ in THF solution, and the relative reactivities of the two silenes are inverted as compared to the situation in hexane or MeCN solution. These effects are due to complexation of the silenes with the ether solvent, the equilibrium constant for which enters the expression for the overall rate constant for reaction. The temperature dependencies of the rate constants in THF are qualitatively similar to those observed previously for reaction of $\mathbf{1} \mathbf{a}, \mathbf{b}$ with acetone, another nucleophilic silene trapping agent of relatively low Bronsted acidity. The relatively large effects of the solvent-silene complexation equilibrium for nonacidic silene traps, as compared to more acidic ones such as alcohols or acetic acid, are due to the fact that the reaction proceeds almost exclusively via the free silene, and the solvent complexes are relatively unreactive. More detailed comparisons suggest that in fact, reaction via the silene-THF complex may contribute in a minor way to the kinetics of $n$-butylamine addition to $\mathbf{1 b}$, the more highly electrophilic (and hence more strongly complexed) of the two silenes studied in this work. The possible intervention of this 
pathway is manifested by a slight rate acceleration at the low end of the temperature range studied, where the fractional concentration of the silene-THF complex is highest and results in a smaller overall activation energy for reaction of this silene with the amine as compared to that for 1a. The opposite trend in activation energy is observed for the reactions of $\mathbf{1 a}, \mathbf{b}$ in THF with the substantially weaker nucleophile, acetone. Evidence for reaction of silene-THF complexes has been reported previously for the reaction of $\mathbf{1 a}, \mathbf{b}$ with alcohols and acetic acid, whose higher proton acidities can lead to reaction of the sileneTHF complex via initial protonation at the silenic carbon. Future work will further explore the effects of silene electrophilicity, solvent complexation, and trapping agent nucleophilicity and acidity on the kinetics and mechanisms of the reactions of silicon-carbon double bonds.

\section{Experimental Section}

${ }^{1} \mathrm{H}$ NMR spectra were recorded in deuteriochloroform or cyclohexane- $d_{12}$ on Bruker AC200 or DRX500 spectrometers and are referenced to tetramethylsilane. Infrared spectra were recorded on a BioRad FTS-40 FT/IR spectrometer and are reported in wavenumbers $\left(\mathrm{cm}^{-1}\right)$. Ultraviolet absorption spectra were recorded on a Varian Cary 50 spectrophotometer. Lowresolution mass spectra were determined by gas chromatography (GC)/mass spectrometry (MS), using a Hewlett-Packard 5890II gas chromatograph equipped with a HP-5971 mass selective detector and a DB-5 fused silica capillary column $(30 \mathrm{~m} \times 0.25$ $\mathrm{mm} ; 0.25 \mu \mathrm{m}$ film; Agilent Technologies). High-resolution electron impact mass spectra and exact masses were determined using a VGH ZABE mass spectrometer.

Analytical gas chromatographic analyses were carried out using a Hewlett-Packard 5890II+ gas chromatograph equipped with a conventional heated splitless injector, flame ionization detector, HP3396A integrator, and DB1 or DB1701 megabore capillary columns $(15 \mathrm{~m} \times 0.53 \mathrm{~mm}$; Chromatographic Specialties, Inc.).

Acetonitrile (Caledon Reagent) was refluxed over calcium hydride (Fisher) for several days, distilled under dry nitrogen, and then cycled three times through a 1 in $\times 6$ in column of neutral alumina (Aldrich), which had been activated by heating under vacuum (ca. 0.05 Torr) at $320^{\circ} \mathrm{C}$ for $10 \mathrm{~h}$ with periodic shaking. THF (Caledon Reagent) was refluxed over molten potassium in a nitrogen atmosphere and distilled. $n$-Butylamine, tert-butylamine, $N, N$-diethylamine, and $N, N, N$-triethylamine (Aldrich) were distilled over solid potassium hydroxide. The 1,1-diarylsilacyclobutanes $(\mathbf{4 a}, \mathbf{b})$ were prepared according to the published methods. ${ }^{18}$

Steady state photolysis $(254 \mathrm{~nm})$ of deoxygenated $0.02-0.08$ M solutions of the 1,1-diarylsilacyclobutanes $\mathbf{4 a}, \mathbf{b}$ (10-20 mg) in hexane or cyclohexane- $d_{12}$ containing a ca. $10 \%$ molar excess of $n-\mathrm{BuNH}_{2}, t-\mathrm{BuNH}_{2}$, or $\mathrm{Et}_{2} \mathrm{NH}$ was carried out using a Rayonet photochemical reactor. Photolyses were carried to 50$95 \%$ conversion, as determined by GC or ${ }^{1} \mathrm{H}$ NMR spectroscopic analysis, and yielded ethylene and a single nitrogen-containing product in each case. The products were isolated in impure form as colorless oils in yields of $40-80 \%$, by microdistillation of the crude, yellow residues obtained from evaporation of solvent and excess amine. They were not purified further because of a sensitivity to hydrolysis and prolonged heating. The products were identified by a combination of GC/MS, IR, ${ }^{1} \mathrm{H}$ and ${ }^{13} \mathrm{C}$ NMR spectroscopy, and high-resolution exact mass determinations on the $\mathrm{M}^{+}$or $\left(\mathrm{M}^{+}-15\right)$ ions, the latter being used in the cases of $\mathbf{5 b}$ and $\mathbf{6 b}$ because the $\mathrm{M}^{+}$ions are extremely weak. The identity of aminosilane 7a was verified by GC coinjection with an authentic sample, which was prepared as follows.
A solution of $n$-butyllithium $(20.5 \mathrm{mmol})$ in anhydrous ether $(50 \mathrm{~mL})$ was placed in a $250 \mathrm{~mL}$ round bottom flask fitted with a condenser, addition funnel, nitrogen inlet, and magnetic stirrer and cooled in an ice bath. A solution of $N, N$-diethylamine $(1.5$ $\mathrm{g}, 20.5 \mathrm{mmol})$ in anhydrous diethyl ether $(30 \mathrm{~mL})$ was then added dropwise with stirring over ca. $30 \mathrm{~min}$. The ice bath was removed, and the solution was stirred for $1 \mathrm{~h}$, after which a solution of diphenylmethylchlorosilane $(4.77 \mathrm{~g}, 20.5 \mathrm{mmol})$ in anhydrous ether $(50 \mathrm{~mL})$ was added dropwise over $30 \mathrm{~min}$, causing the formation of a colorless precipitate. The mixture was stirred for a further $3 \mathrm{~h}$ and filtered, and the solvent was removed by distillation. Pentane $(20 \mathrm{~mL})$ was added to the resulting yellow oily solid, the mixture was filtered, and the solvent was removed again by distillation to yield a yellow oil, which was then distilled under vacuum. The product was collected as a colorless oil (bp 97-98 ${ }^{\circ} \mathrm{C}(0.03 \mathrm{mmHg}), 4.85$ $\mathrm{g}, 87.5 \%)$ and identified as ( $N, N$-diethylamino)methyldiphenylsilane (7a) on the basis of the following spectroscopic data. ${ }^{1} \mathrm{H} \mathrm{NMR}\left(\mathrm{CDCl}_{3}\right): \delta(\mathrm{ppm}) 7.56(\mathrm{~m}, 4 \mathrm{H}), 7.36(\mathrm{~m}, 6 \mathrm{H}), 2.91$ $(\mathrm{q}, 4 \mathrm{H}), 0.99(\mathrm{t}, 6 \mathrm{H}), 0.58(\mathrm{~s}, 3 \mathrm{H}) .{ }^{13} \mathrm{C} \mathrm{NMR}\left(\mathrm{CDCl}_{3}\right): \delta(\mathrm{ppm})$ 138.1, 134.8, 129.1, 127.6, 40.1, 15.5, -2.2. IR (neat): 3069 (m), 2965 (s), 2866 (m), 1428 (m), 1375 (m), 1253 (m), 1172 (s), 1110 (s), 1028 (s), 927 (m), 789 (s), 724 (m), 700 (m). EIMS: m/z (I) 269 (7), 255 (14), 254 (62), 198 (19) 197 (100), 183 (8), 181 (9), 121 (15), 105 (20). HRMS: $\mathrm{m} / \mathrm{z}$ calcd for $\mathrm{C}_{17} \mathrm{H}_{23} \mathrm{NSi}\left(\mathrm{M}^{+}\right), 269.1600$; found, 269.1586. The ${ }^{13} \mathrm{C} \mathrm{NMR}$ spectrum of the compound is similar to that reported previously by Filleux-Blanchard and An. ${ }^{27}$ Spectroscopic data for the other aminosilanes $(\mathbf{5 a}, \mathbf{6 a}, \mathbf{5 b}-\mathbf{7 b})$ are listed in the Supporting Information.

Nanosecond laser flash photolysis experiments employed the pulses (248 nm; 15-20 ns; 70-120 mJ) from a Lambda Physik Compex 100 excimer laser, filled with $\mathrm{F}_{2} / \mathrm{Kr} / \mathrm{Ne}$ mixtures, and a Luzchem Research mLFP-111 laser flash photolysis system. Solutions were prepared at concentrations such that the absorbance at the excitation wavelength $(248 \mathrm{~nm})$ was ca. $0.7(0.003-$ $0.004 \mathrm{M}$ ) and was flowed continuously through a $7 \times 7$ Suprasil flow cell connected to a calibrated $100 \mathrm{~mL}$ reservoir. Oxygen has no discernible effect on the lifetimes of $\mathbf{1 a}, \mathbf{b},{ }^{18}$ so the solutions were not deoxygenated. Solution temperatures were measured with a Teflon-coated copper/constantan thermocouple inserted directly into the flow cell. Reagents were added to the reservoir by microliter syringe as aliquots of standard solutions. Rate constants were calculated by linear least-squares analysis of decay rate-concentration data (6-10 points) that spanned at least a factor of 5 (usually more than 1 order of magnitude) in the transient decay rate. Errors are quoted as twice the standard deviation obtained from the least-squares analysis in each case.

Acknowledgment. We thank the Natural Sciences and Engineering Research Council of Canada for financial support, Cameron R. Harrington for the preparation of the authentic sample of $\mathbf{7 a}$, and a referee for insightful comments.

Supporting Information Available: Spectroscopic data for aminosilanes 5-7, plots of $\left(k_{\text {decay }}-k_{0}\right)$ vs [amine] for the reaction of $\mathbf{1 b}$ with $n-\mathrm{BuNH}_{2}, t-\mathrm{BuNH}_{2}$, and $\mathrm{Et}_{2} \mathrm{NH}$ in hexane and $\mathrm{MeCN}$ solution at $23{ }^{\circ} \mathrm{C}$, and tables of absolute rate constants for reaction of $\mathbf{1 a}, \mathbf{b}$ with the three amines in hexane, $\mathrm{MeCN}$, and THF at various temperatures between 0 and $60^{\circ} \mathrm{C}$. This material is available free of charge via the Internet at http://pubs.acs.org. 


\section{References and Notes}

(1) Wiberg, N. J. Organomet. Chem. 1984, 273, 141-177.

(2) Wiberg, N.; Kopf, H. J. Organomet. Chem. 1986, 315, 9-18.

(3) Wiberg, N.; Kim, C.-K. Chem. Ber. 1986, 119, 2980-2994.

(4) Wiberg, N.; Joo, K. S.; Polborn, K. Chem. Ber. 1993, 126, 67-69.

(5) Baines, K. M.; Stibbs, W. G. Adv. Organomet. Chem. 1996, 39, 275-324.

(6) Brook, A. G.; Brook, M. A. Adv. Organomet. Chem. 1996, 39 , $71-158$

(7) Leigh, W. J. Pure Appl. Chem. 1999, 71, 453-462.

(8) Brook, A. G.; Yu, Z. Organometallics 2000, 19, 1859-1863.

(9) Morkin, T. L.; Owens, T. R.; Leigh, W. J. Kinetic studies of the reactions of $\mathrm{Si}=\mathrm{C}$ and $\mathrm{Si}=\mathrm{Si}$ double bonds. In The Chemistry of Organosilicon Compounds; Rappoport, Z., Apeloig, Y., Eds.; John Wiley and Sons: New York, 2001; Vol. 3, pp 949-1026.

(10) Sakurai, H. Mechanism and structures in alcohol addition reactions of disilenes and silenes. In The Chemistry of Organic Silicon Compounds; Rappoport, Z., Apeloig, Y., Eds.; Wiley and Sons Ltd: New York, 1998; pp 827-855.

(11) Morkin, T. L.; Leigh, W. J. Acc. Chem. Res. 2001, 34, 129-136.

(12) Wiberg, N.; Wagner, G.; Muller, G.; Riede, J. J. Organomet. Chem. 1984, 271, 381-391.

(13) Kira, M.; Maruyama, T.; Sakurai, H. J. Am. Chem. Soc. 1991, 113, 3986-3987.

(14) Leigh, W. J.; Sluggett, G. W. J. Am. Chem. Soc. 1994, 116, 1046810476

(15) Leigh, W. J.; Bradaric, C. J.; Kerst, C.; Banisch, J. H. Organometallics 1996, 15, 2246-2253.
(16) Sluggett, G. W.; Leigh, W. J. J. Am. Chem. Soc. 1992, 114, 11951201.

(17) Leigh, W. J.; Bradaric, C. J.; Sluggett, G. W.; Venneri, P.; Conlin, R. T.; Dhurjati, M. S. K.; Ezhova, M. B. J. Organomet. Chem. 1998, 561 , 19-27.

(18) Bradaric, C. J.; Leigh, W. J. Can. J. Chem. 1997, 75, 1393-1402.

(19) Leigh, W. J.; Li, X. Organometallics 2002, 21, 1197-1207.

(20) Leigh, W. J.; Bradaric, C. J.; Morkin, T. L.; Li, X. Organometallics 2001, 20, 932-936.

(21) Bradaric, C. J.; Leigh, W. J. Organometallics 1998, 17, 645-651.

(22) CRC Handbook of Chemistry and Physics; CRC Press: Boca Raton, 1995; pp 6-241.

(23) Izutsu, K. Acid-Base Dissociation Constants in Dipolar Aprotic Solvents; IUPAC Chemical Data Series No. 35; Blackwell Scientific Publications: Oxford, 1990; pp 17-35.

(24) We have previously stated that the maximum rate constant is obtained at the temperature where the partitioning ratio is approximately equal to 0.5 (i.e., when $\left.k_{-\mathrm{C}} \approx k_{-\mathrm{H}}\right)^{9,11,21}$ This may be the case for the addition of methanol to silene $\mathbf{1 b}$ in acetonitrile solution ${ }^{18}$ but is not necessarily so in general.

(25) The other extreme situation where such behavior would be obtained is one in which the complex partitioning ratios for the two derivatives are similar and vary similarly with temperature, but the activation energy for the initial step is higher for the more reactive compound $(\mathbf{1 b})$. This seems unlikely but nevertheless cannot be ruled out on the basis of the data at hand.

(26) Espenson, J. H. Chemical Kinetics and Reaction Mechanisms; McGraw-Hill: New York, 1995; pp 15-45.

(27) Filleux-Blanchard, M. L.; An, N. D. Org. Magn. Res. 1979, 12, 